\title{
Vision of correction for classic homocystinuria
}

\author{
Dwight D. Koeberl
}

Department of Pediatrics, Division of Medical Cenetics, Duke University Medical Center, Durham, North Carolina, USA

\begin{abstract}
Inherited metabolic disorders are often characterized by the lack of an essential enzyme and are currently treated by dietary restriction and other strategies to replace the substrates or products of the missing enzyme. Patients with homocystinuria lack the enzyme cystathionine $\beta$-synthase (CBS), and many of these individuals do not respond to current treatment protocols. In this issue of the $\mathrm{JCl}$, Bublil and colleagues demonstrate that enzyme replacement therapy (ERT) provides long-term amelioration of homocystinuria-associated phenotypes in CBS-deficient murine models. A PEGylated form of CBS provided long-term stability and, when used in conjunction with the methylation agent betaine, dramatically increased survival in mice fed a normal diet. The results of this study provide one of the first examples of ERT for a metabolic disorder and suggest that PEGylated CBS should be further explored for use in patients.
\end{abstract}

\section{Modified CBS for}

homocystinuria ERT

In this issue, Bublil and colleagues provide a new example of ERT (5). Specifically, they show that treating classic homocystinuria requires modification of the CBS amino acid sequence and PEGylation (conjugation with PEG) of the enzyme. Moreover, this ERT strategy achieved a more stable correction of homocystinuria in a CBSdeficient mouse model than did replacement with nonmodified CBS. Homocystinuria mice treated with PEGylated CBS exhibited a $75 \%$ reduction in homocysteinemia and normalization of cysteine (Figure 1C). This impressive reduction suggests that use of the PEGylated CBS has promise to relieve the risk for thrombosis and to potentially reduce the risk for lens subluxation in patients detected by newborn screening.

The work of Bublil et al. represents a feat of bioengineering that has addressed several hurdles to ERT with CBS (5). PEGylation of CBS stabilized the subcutaneously administered enzyme and led to a uniform reduction in plasma homocysteine levels in the treated mice. In contrast, unmodified CBS resulted in a variable and reduced effect. The plasma cystathionine concentration increased in correlation with decreased homocysteine in PEGylated CBS-treated mice, confirming that the homocysteinelowering effect was from CBS activity. Homocysteine was reduced in target tissues, including liver, kidney, and brain, demonstrating systemic correction following subcutaneous administration of PEGylated CBS. The addition of a second modification, mutation of the highly reactive cysteine at position 15 to serine (CBS $\mathrm{C} 15 \mathrm{~S})$, reduced the formation of CBS aggregates and promoted more uniform PEGylation of the recombinant enzyme. Evaluation of double-modified CBS, the methylation agent betaine, and the two treatments in combination revealed that both PEGylated CBS C15S and betaine independently reduced homocysteine in CBS-deficient mice; however, together,
Conflict of interest: The author has received research grants from Cenzyme Corporation and Roivant Sciences. He has applied for and been awarded patents covering treatments for Pompe disease (US Patent Application nos. 10/159,546, 12/801,404, and 13/253,005; European Patent no. 1587923 B1).

Reference information: J Clin Invest. 2016;126(6):2043-2044. doi:10.1172/JCI88251. 
A Normal

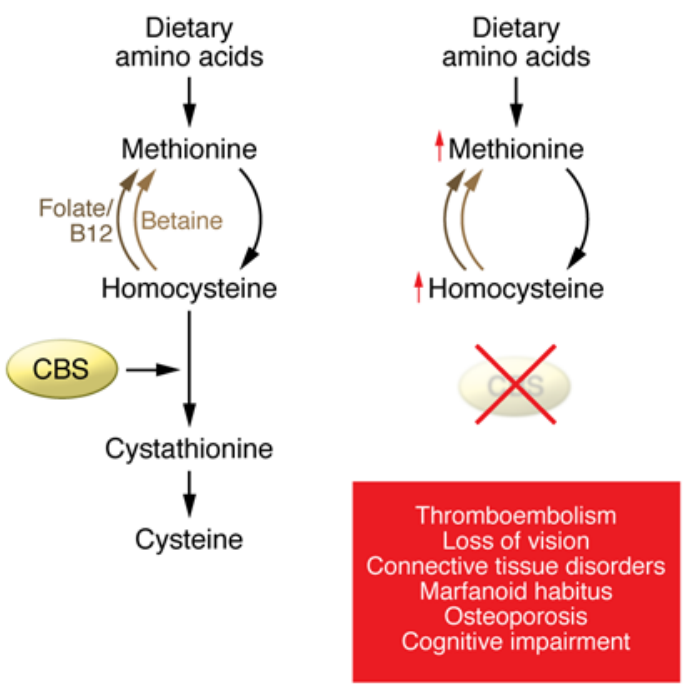

C ERT

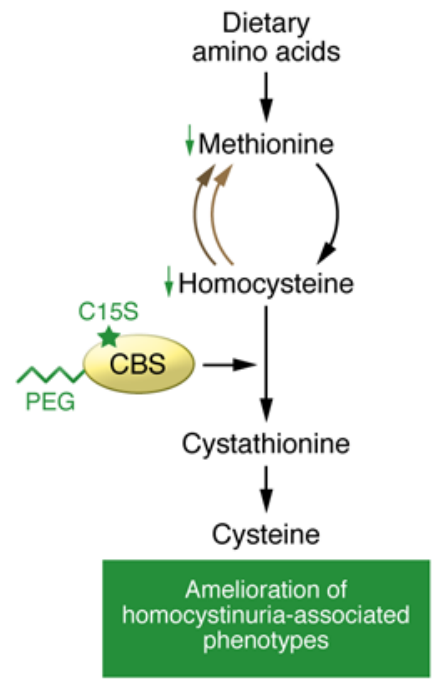

Figure 1. ERT for homocystinuria. (A) In healthy individuals, methionine from dietary sources is metabolized into homocysteine, which is then converted into cysteine by the enzyme CBS. (B) Homocystinuria develops in individuals lacking CBS. Elevated homocysteine results in a variety of adverse effects, including thromboembolism, connective tissue disorders, Marfanoid habitus, osteoporosis, and cognitive impairment. Homocysteine and methionine are elevated to extremely high concentrations. (C) In this issue, Bublil and colleagues developed a strategy for ERT (5). In a murine model of homocystinuria, a PEGylated form of CBS provided long-term reduction of homocysteine levels. Moreover, the introduction of a C15S mutation reduced aggregation of the recombinant, PEGylated enzyme.

the two treatments had synergistic effects and sustained the homocysteine reduction over a 3-week period. Finally, ERT with PEGylated CBS C15S prevented mortality and partially reversed hepatosteatosis, confirming the efficacy of this strategy in the mouse model.

\section{Conclusions and future directions}

The results of the study by Bublil and colleagues indicate that ERT has potential as an effective treatment for individuals with so-called nonresponsive homocystinuria that fails to respond to pyridoxine supplementation and leads to developmental disability (6). The clinical relevance for an ERT strategy to treat nonresponsive individuals is great, given the severity of this form of the disease, which presents with earlier-onset lens subluxation and severe myopia (7). The treatment goal of reduced plasma homocysteine concentrations is critical for preventing homocystinuria-associated complications, including the recurrence of thrombosis and stroke (8). Betaine alone may be effective in nonresponsive patients for whom dietary therapy has been ineffective (9), and therefore ERT might not be needed for every nonresponsive patient.

More work will need to be done before ERT for homocystinuria is used in the clinic. Even if no unanticipated toxicities of PEGylated CBS are revealed, there are several limitations to the use of ERT in patients. ERT requires frequent injections, albeit these could be self-administered subcutaneously. Patients on ERT are also at risk for developing hypersensitivity and neutralizing immune responses against the recombinant enzyme. Finally, the cost of these treatments is likely to remain high (10). However, the success of ERT for homocystinuria also forecasts the development of gene therapy such as that underway for lysosomal storage disorders to address these limitations (11). Overall, the development of ERT for homocystinuria holds great promise for the treatment of this disorder and other inher- ited disorders of amino acid metabolism, especially in combination with newborn screening and presymptomatic treatment.

\section{Acknowledgments}

The author was supported in part through the Alice and Y.T. Chen Pediatric Genetics and Genomics Center.

Address correspondence to: Dwight D. Koeberl, Department of Pediatrics/Division of Medical Genetics, Duke University Medical Center, Durham, North Carolina 27705, USA. Phone: 919.681.9919; E-mail: dwight.koeberl@duke.edu.

1. Frazier DM, et al. The tandem mass spectrometry newborn screening experience in North Carolina: 1997-2005. JInherit Metab Dis. 2006;29(1):76-85.

2. Desnick RJ. Enzyme replacement and enhancement therapies for lysosomal diseases. J Inherit Metab Dis. 2004;27(3):385-410.

3. Yap S, Naughten E. Homocystinuria due to cystathionine beta-synthase deficiency in Ireland: 25 years' experience of a newborn screened and treated population with reference to clinical outcome and biochemical control. J Inherit Metab Dis. 1998;21(7):738-747.

4. Mudd SH, Finkelstein JD, Irreverre F, Laster L. Homocystinuria: an enzymatic defect. Science. 1964;143(3613):1443-1445.

5. Bublil EM, et al. Enzyme replacement with PEGylated cystathionine $\beta$-synthase ameliorates homocystinuria in murine model. J Clin Invest. 2016;126(6):2372-2384.

6. Picker JD, Levy HL. Homocystinuria caused by cystathionine beta-synthase deficiency. In: Pagon RA, et al., eds. GeneReviews(R) [Internet] Seattle, Washington, USA: University of Washington, Seattle; 1993-2016.

7. Mulvihill A, Yap S, O'Keefe M, Howard PM, Naughten ER. Ocular findings among patients with late-diagnosed or poorly controlled homocystinuria compared with a screened, well-controlled population. JAAPOS. 2001;5(5):311-315.

8. Lawson-Yuen A, Levy HL. The use of betaine in the treatment of elevated homocysteine. Mol Genet Metab. 2006;88(3):201-207.

9. Singh RH, Kruger WD, Wang L, Pasquali M, Elsas LJ. Cystathionine beta-synthase deficiency: effects of betaine supplementation after methionine restriction in B6-nonresponsive homocystinuria. Genet Med. 2004;6(2):90-95.

10. Banugaria SG, et al. The impact of antibodies on clinical outcomes in diseases treated with therapeutic protein: lessons learned from infantile Pompe disease. Genet Med. 2011;13(8):729-736.

11. Koeberl DD, Kishnani PS. Immunomodulatory gene therapy in lysosomal storage disorders. Curr Gene Ther. 2009;9(6):503-510. 